\title{
Metformin-Associated Lactic Acidosis: A Case Report
}

\author{
Takehide Umeda $^{1} \cdot$ Taro Minami $^{2} \cdot$ Keith Bartolomei $^{2} \cdot$ Eleanor Summerhill $^{3}$
}

Published online: 9 February 2018

(C) The Author(s) 2018. This article is an open access publication

\begin{abstract}
A 54-year-old woman with type 2 diabetes mellitus, hypertension, and peripheral vascular disease developed life-threatening lactic acidosis during treatment with metformin for type 2 diabetes. The woman received metformin at $1000 \mathrm{mg}$ orally twice a day for type 2 diabetes. She presented to our emergency department with a 3-day history of severe watery diarrhea, nausea, and vomiting. Her grandson whom she cared for had gastroenteritis several days prior to the onset of her symptoms. She was confused and hypotensive with a blood pressure of $70 / 39 \mathrm{mmHg}$. Her initial laboratory findings were remarkable with an arterial blood gas $\mathrm{pH}$ 6.57, $\mathrm{HCO}_{3}^{-}$ $2 \mathrm{mEq} / \mathrm{L}$, anion gap $30 \mathrm{mmol} / \mathrm{L}$, and lactate $16.3 \mathrm{mmol} / \mathrm{L}$. She was diagnosed with severe lactic acidosis. Metformin was discontinued. Upon arrival in the emergency department, she became unresponsive and experienced a pulseless electrical activity cardiac arrest. After resuscitation, her severe acidemia persisted despite aggressive intervention with volume resuscitation and vasopressors, leading to the initiation of renal replacement therapy. After multiple dialysis treatments, her severe acidemia resolved. Serum metformin concentration from presentation ultimately returned to $42 \mathrm{mcg} / \mathrm{mL}$ (therapeutic concentration: 1-2 $\mathrm{mcg} / \mathrm{mL}$ ). She was discharged from the hospital on day 15
\end{abstract}

Takehide Umeda

umeda006@umn.edu

1 Division of Pulmonary, Allergy, Critical Care, and Sleep Medicine, University of Minnesota, 420 Delaware Street SE, MMC 276, Minneapolis, MN 55455, USA

2 Memorial Hospital of Rhode Island, The Warren Alpert Medical School of Brown University, Pawtucket, RI, USA

3 Department of Pulmonary and Critical Care Medicine, Lahey Hospital and Medical Center, Burlington, MA, USA without any neurologic complications. A Naranjo assessment score of 8 was obtained, indicating a probable relationship between the patient's lactic acidosis and her use of the suspect drug.

\section{Key Points}

Metformin toxicity should be considered in any patient with type 2 diabetes mellitus presenting with severe lactic acidosis and profound acidemia.

Metformin-associated lactic acidosis can be treated with renal replacement therapy and generally has a higher survival rate than other causes of severe lactic acidosis.

Metformin should be used with caution in patients with chronic kidney disease. Education of patients with type 2 diabetes and their healthcare providers is crucial to avoid metformin-associated lactic acidosis when there is a risk of renal failure.

\section{Introduction}

Metformin is an oral hypoglycemic medication commonly used as a first-line agent to treat type 2 diabetes mellitus owing to its favorable safety profile. A recent study revealed that even in patients with mild-to-moderate chronic kidney disease, drug concentrations generally remain within the therapeutic range and lactate concentrations are not substantially increased [1]. 
However, the situation is different when a patient has acute renal insufficiency with progressively worsening kidney function, hepatic insufficiency, or acute infection as build-up of its serum concentration can lead to profound life-threating lactic acidosis. We present a case of metformin-associated lactic acidosis (MALA), which resulted in cardiopulmonary arrest. Treatment of MALA consists of supportive therapy including mechanical ventilation and vasopressors. In severe cases, renal replacement therapies, such as hemodialysis or continuous veno-venous hemofiltration, are used for drug removal and correction of severe acidemia [2, 3].

\section{Case Report}

A 54-year-old Hispanic woman with type 2 diabetes, hypertension, and peripheral vascular disease status post a right-foot transmetatarsal amputation 3 years prior presented to our hospital with a 3-day history of severe watery diarrhea, nausea, vomiting, and mental status change. She reported taking all of her home oral medications including lisinopril $40 \mathrm{mg}$ daily, metoprolol succinate $50 \mathrm{mg}$ daily, pravastatin $40 \mathrm{mg}$ daily, aspirin $81 \mathrm{mg}$ daily, hydrochlorothiazide $12.5 \mathrm{mg}$ daily, and metformin $1000 \mathrm{mg}$ twice a day. She also took 50 units of insulin detemir by subcutaneous injection every night. She had not taken 30 units of insulin lispro by subcutaneous injection before each meal for about $24 \mathrm{~h}$ because of poor oral intake. She reported that she was barely able to eat or drink because of nausea and appetite loss.

In the emergency department, she was found to be hypoglycemic with a glucose level of $47 \mathrm{mg} / \mathrm{dL}$. Glucagon was administered. Initially, she was able to talk, but was confused and hypotensive with a blood pressure of 70/ $39 \mathrm{mmHg}$. Physical examination was remarkable for disorientation, dry oral mucosa, and decreased bowel sounds. Otherwise physical examination was unremarkable, with normal chest and heart examinations. Soon after arrival, the patient became unresponsive and went into a cardiopulmonary arrest with pulseless electrical activity. Cardiopulmonary resuscitation, including chest compression and endotracheal intubation with mechanical ventilation, was initiated. She regained spontaneous circulation after 3 min of cardiopulmonary resuscitation and was transferred to the medical intensive care unit for further care. After the transfer to the intensive care unit, her blood pressure was $98 / 37 \mathrm{mmHg}$ on $0.3 \mathrm{mcg} / \mathrm{kg} / \mathrm{min}$ of norepinephrine with profound acidemia. Laboratory findings were remarkable with arterial $\mathrm{pH} 6.57, \mathrm{HCO}_{3}^{-} 2 \mathrm{mEq} / \mathrm{L}$, anion gap $30 \mathrm{mmol} / \mathrm{L}$, osmolar gap of 21 , lactate $16.3 \mathrm{mmol} / \mathrm{L}$, glycosylated hemoglobin $7.3 \mathrm{mg} / \mathrm{dL}$, and creatinine of $8.07 \mathrm{mg} / \mathrm{dL}$. Her baseline creatinine was in the normal range. Two months prior to admission, her serum creatinine was $0.86 \mathrm{mg} / \mathrm{dL}$. A computed tomography scan of the abdomen and pelvis was performed to look for a possible source of infection. The result was unrevealing except for non-specific gallbladder wall thickening. Vasopressin infusion was added later to maintain her mean arterial pressure above $60 \mathrm{mmHg}$.

The differential diagnosis for the anion gap acidosis was broad. Originally, it was felt that her hypotension, acidemia, and severe metabolic derangement could be consistent with severe sepsis and septic shock. An acute coronary event leading to cardiogenic shock was also considered in the setting of her cardiac arrest. However, given the severity of the acidemia ( $\mathrm{pH}$ 6.57) and the persistence of lactic acidosis despite aggressive interventions and normalization of blood pressure, metformin toxicity became the most likely diagnosis. This diagnosis was strengthened by the fact that she had likely been experiencing impaired renal function prior to her presentation given the hypovolemia, hypotension, and concomitant angiotensin-converting enzyme inhibitor use, which was presumed to have led to impaired clearance of the drug.

After cardiopulmonary resuscitation, the patient required continued mechanical ventilation for type IV respiratory failure as a result of shock and acidemia. She required norepinephrine, and vasopressin for persistent shock. As the degree of acidemia was considered to be out of proportion with possible sepsis, metformin toxicity was suspected early in the course of the hospitalization. Urgent renal replacement therapy with intermittent hemodialysis was started. After initiation of hemodialysis, the severe acidemia resolved. With normalization of the acid base status, hemodynamics gradually started to improve and required a reduced dose of norepinephrine and vasopressin infusion.

The work-up for infectious etiologies including blood and stool cultures was negative. In the setting of cardiac arrest, cardiac biomarkers were checked and an elevated troponin of $4.42 \mathrm{ng} / \mathrm{mL}$ was identified. This troponin leak was considered to represent demand ischemia in the setting of stable coronary artery disease and profound metabolic and post-resuscitative hemodynamic stress. Serial electrocardiograms were negative for significant ST-T wave changes. An echocardiogram conducted on day 2 revealed a relatively preserved ejection fraction of $50 \%$ without regional wall motion abnormalities.

Norepinephrine and vasopressin were discontinued on day 4. The patient was extubated on day 6. Serum metformin concentration returned to $42 \mathrm{mcg} / \mathrm{mL}$ (therapeutic concentration: $1-2 \mathrm{mcg} / \mathrm{mL}$ ) on day 7 . She was discharged from the intensive care unit on day 9, and was discharged from the hospital on day 15, without any neurological 
complications from the cardiac arrest. Dialysis was discontinued within 1 month of discharge as her renal function returned to baseline.

The patient later reported that her grandson whom she cared for had gastroenteritis several days prior to the onset of her symptoms. After discharge, the patient was evaluated by a nuclear stress test, which was abnormal. Subsequently, the coronary angiography was performed. This revealed three-vessel disease with a $90 \%$ stenosis in the mid-left anterior descending artery, $80 \%$ stenosis in the first obtuse marginal artery, and a $90 \%$ stenosis in the midright coronary artery. She underwent a coronary artery bypass graft without any complications and has been doing well since then.

\section{Discussion}

Metformin is commonly used and widely considered to be safe. Nevertheless, MALA is a well-known side effect of the medication. Findings of a recent meta-analysis did not support a strong association of MALA with metformin in patients with stable chronic kidney disease [1]. This finding has also been supported by other studies [4]. However, patients with significant renal impairment, whether from advanced chronic kidney disease or acute kidney injury, remain at high risk for complications from this medication $[5,6]$. Metformin is excreted from the proximal tubules of the kidneys without being metabolized. Thus, metformin accumulates in renal failure [6]. Lactic acidosis resulting from metformin toxicity should be suspected in any patient who has all of the following five criteria: (1) a history of metformin administration; (2) a markedly elevated lactate level $(>15 \mathrm{mmol} / \mathrm{L})$ with a large anion gap $(>20 \mathrm{mmol} /$ L); (3) severe acidemia ( $\mathrm{pH} 7.1)$; (4) a very low serum bicarbonate level $(<10 \mathrm{mmol} / \mathrm{L})$; and (5) a history of renal insufficiency (glomerular filtration rate $<45 \mathrm{~mL} / \mathrm{min}$ or serum creatinine level $>2.0 \mathrm{mg} / \mathrm{dL}$ ) [7].

Patients with diabetes commonly have coronary artery disease. Extreme acidosis reduces ventricular contractility and cardiac output [7]. In the setting of hypotension and severe coronary artery disease, our patient's coronary perfusion was likely critically reduced. This severe reduction in coronary perfusion combined with profound acidemia is likely what led to pulseless electrical activity arrest. Her significantly impaired circulation in the setting of severe peripheral vascular disease could have worsened the increased lactate production in peripheral tissues.

In a previous study by Friesecke et al., overall survival rates for patients with an arterial blood $\mathrm{pH}$ of $<7.00$ was significantly better (50 vs. $0 \%$ ) in MALA cases when compared with other causes of lactic acidosis [8]. This is mainly owing to the fact that MALA is treatable with aggressive interventions, including dialysis. The overall mortality rate of MALA is $25.4 \%$ according to the study by Renda et al. [2]. Mortality was associated with the extent of acidosis while acute renal failure was associated with a low mortality. Non-surviving cases tend to have multiple comorbidities other than renal failure, which is treatable by dialysis [2]. In our particular case, coexisting severe coronary artery disease and already impaired circulation by severe peripheral artery disease are considered to have contributed to her severe accumulation of lactic acid, hypotension, and subsequent cardiopulmonary arrest with pulseless electrical activity.

With regard to renal replacement therapy for MALA treatment, a recent study revealed that the clearance of metformin by continuous veno-venous hemofiltration was less than that generally reported to occur with conventional hemodialysis. Thus, continuous veno-venous hemofiltration should be considered only in patients who are too hemodynamically unstable to tolerate hemodialysis [3,9]. Indications for extracorporeal treatment include lactate $>20 \mathrm{mmol} / \mathrm{L}, \mathrm{pH} \mathrm{7.0,} \mathrm{shock,} \mathrm{failure} \mathrm{of} \mathrm{standard} \mathrm{sup-}$ portive measures, and a decreased level of consciousness [9].

Vomiting and diarrhea out of the ordinary are the first signs of MALA [6]. Metformin toxicity may mimic sepsis with gastrointestinal symptoms because severe acidemia has been documented to cause nausea, vomiting, abdominal pain, and leukocytosis. It was also misdiagnosed as mesenteric ischemia owing to significant lactic acidosis with gastrointestinal symptoms [10]. This lactic acidosis with gastrointestinal symptoms can lead clinicians to a misdiagnosis and subsequent mortality without recognizing it as MALA. Thus, potentially, there could be many underdiagnosed MALA cases in the real world. If the diagnosis of metformin toxicity is not entertained early in the presentation, providers may miss the critical window in which definitive treatment can be lifesaving. The true incidence of MALA has been controversial. In a Cochrane review, it was estimated that there were no cases of fatal or non-fatal lactic acidosis in 70,490 patient-years of metformin use [11]. In the most recent original study estimating the incidence of MALA conducted by Haloob et al., the incidence of MALA was actually estimated at 19.46 per 100,000 patient-year exposures to metformin. They also found the relative risk of lactic acidosis in patients taking metformin was $13.53 \quad(95 \%$ confidence interval 7.88-21.66) compared with the general population [12]. In this study, all cases were associated with acute kidney injury. 


\section{Conclusion}

This case highlights a relatively rare, though important complication from metformin-MALA. Gastroenteritis, which is just severe enough to create acute kidney injury, could progress into a life-threatening MALA, as seen in our case. It is crucial to maintain a high suspicion of this complication in the differential diagnosis at the earliest stage for patients with metabolic acidosis and a history of metformin use. If we fail to check the level at the earliest stage, we may miss the chance of diagnosis. Severe metabolic acidosis may be treated with renal replacement therapy regardless of the underlying causes, and because metformin is dialyzable, this condition may still be underdiagnosed. Education of both patients and providers to avoid metformin when there is a risk of renal failure, particularly with hypovolemia, is crucial to prevent MALA.

Funding No sources of funding were received for the preparation of this article.

\section{Compliance with Ethical Standards}

Conflict of interest Takehide Umeda, Taro Minami, Keith Bartolomei, and Eleanor Summerhill have no conflicts of interest directly relevant to the content of this article.

Consent to participate Written informed consent was obtained from the patient for publication of this case report. A copy of the written consent may be requested for review from the corresponding author.

Open Access This article is distributed under the terms of the Creative Commons Attribution-NonCommercial 4.0 International License (http://creativecommons.org/licenses/by-nc/4.0/), which permits any noncommercial use, distribution, and reproduction in any medium, provided you give appropriate credit to the original author(s) and the source, provide a link to the Creative Commons license, and indicate if changes were made.

\section{References}

1. Inzucchi SE, Lipska KJ, Mayo H, Bailey CJ, McGuire DK. Metformin in patients with type 2 diabetes and kidney disease: a systematic review. JAMA. 2014;312(24):2668-75.

2. Renda F, Mura P, Finco G, Ferrazin F, Pani L, Landoni G. Metformin-associated lactic acidosis requiring hospitalization: a national 10 year survey and a systematic literature review. Eur Rev Med Pharmacol Sci. 2013;17(Suppl. 1):45-9.

3. Nguyen HL, Concepcion L. Metformin intoxication requiring dialysis. Hemodial Int. 2011;15(Suppl. 1):S68-71.

4. Adam WR, O'Brien RC. A justification for less restrictive guidelines on the use of metformin in stable chronic renal failure. Diabet Med. 2014;31(9):1032-8.

5. Vecchio S, Protti A. Metformin-induced lactic acidosis: no one left behind. Crit Care. 2011;15(1):107.

6. Duong JK, Furlong TJ, Roberts DM, Graham GG, Greenfield JR, Williams KM, Day RO. The role of metformin in metforminassociated lactic acidosis (MALA): case series and formulation of a model of pathogenesis. Drug Saf. 2013;36(9):733-46.

7. Protti A, Gattinoni L. Case 23-2013: a 54-year-old woman with metformin toxicity. N Engl J Med. 2013;369(18):1768-9.

8. Friesecke S, Abel P, Roser M, Felix SB, Runge S. Outcome of severe lactic acidosis associated with metformin accumulation. Crit Care. 2010;14(6):R226.

9. Calello DP, Liu KD, Wiegand TJ, Roberts DM, Lavergne V, Gosselin S, Hoffman RS, Nolin TD, Ghannoum M. Extracorporeal treatment for metformin poisoning: systematic review and recommendations from the Extracorporeal Treatments in Poisoning Workgroup. Crit Care Med. 2015;43(8):1716-30.

10. Ncomanzi D, Sicat RM, Sundararajan K. Metformin-associated lactic acidosis presenting as an ischemic gut in a patient who then survived a cardiac arrest: a case report. J Med Case Rep. 2014;8:159.

11. Salpeter SR, Greyber E, Pasternak GA, Salpeter EE. Risk of fatal and nonfatal lactic acidosis with metformin use in type 2 diabetes mellitus. Cochrane Database Syst Rev. 2010;(4):CD002967.

12. Haloob I, de Zoysa JR. Metformin associated lactic acidosis in Auckland City Hospital 2005 to 2009. World J Nephrol. 2016;5(4):367-71. 